\title{
Point-Counterpoint: Perioperative Cardiac Biomarkers
}

\author{
Shannon Ruzycki, MD, FRCPC and Michael Prystajecky, MD, MSc, FRCPC
}

\author{
About the Authors \\ Shannon Ruzycki, Clinical Lecturer, Department of Medicine, Cumming School of Medicine, University of Calgary \\ Michael Prystajecky, MD, MSc, FRCPC, Assistant Professor, Department of Medicine, University of Saskatchewan \\ Corresponding Author michael.prystajecky@usask.ca \\ Submitted: February 12, 2019. Accepted: April 24, 2019. Published: November 19, 2019. DOI: 10.22374/cjgim.v14i4.347
}

\begin{abstract}
In the following review article, we present arguments for and against the use of postoperative troponin surveillance and preoperative natriuretic peptide testing. This article covers the evidence that informed the CCS 2016 perioperative guidelines and research published since. This review is based on the debate held at the Canadian Society of Internal Medicine/ American College of Physicians Rocky Mountain Chapter Annual Meeting (CSIM/ACP RM) held in October 2018 in Banff, Alberta.
\end{abstract}

\section{Resume}

Dans l'article de synthèse qui suit, nous présentons des arguments pour et contre l'utilisation de la surveillance postopératoire de la troponine et des tests préopératoires de peptides natriurétiques. Cet article porte sur les données probantes qui ont éclairé les lignes directrices périopératoires de la SCC 2016 et les recherches publiées depuis lors. Cet examen est fondé sur le débat qui a eu lieu à l’assemblée annuelle de la section des Rocheuses de la Société canadienne de médecine interne et de l'American College of Physicians (CSIM/ACP RM) tenue en octobre 2018 à Banff, en Alberta.

A total of 1,500,000 inpatient surgeries are performed in Canada each year. ${ }^{1,2}$ In the 30 -days following surgery, $5 \%$ of patients will have a myocardial infarction, $1 \%$ will have a stroke, and 1-2\% will die. ${ }^{3-6}$ Many of these patients will require readmission to hospital. ${ }^{7}$ Cardiovascular complications are therefore a leading cause of postoperative morbidity, mortality, and healthcare expenditures.

The goal of perioperative medicine is to identify patients at high risk of postoperative complications and to mitigate this risk by optimizing medical and surgical care, monitoring patients postoperatively, and managing complications. While previous perioperative guidelines from the American Heart Association (AHA) emphasized functional assessment and pharmacologic stress testing to estimate the postoperative risk of myocardial infarction (MI) and death, ${ }^{8}$ recent guidelines from the Canadian Cardiovascular Society (CCS) instead emphasize the role of cardiac biomarkers for risk stratification. ${ }^{9}$ However, a poll of Canadian Society of Internal Medicine Annual Meeting attendees suggests that uptake of these recommendations has been limited (Figures 1 and Figure 2).

In the following review article, we present arguments for and against the use of postoperative troponin surveillance and preoperative natriuretic peptide testing. This article covers the evidence that informed the CCS 2016 perioperative guidelines and research published since then. This review is based on the debate held at the Canadian Society of Internal Medicine/American College of Physicians Rocky Mountain Chapter Annual Meeting (CSIM/ACP RM) held in October 2018 in Banff, Alberta.

\section{Point: Internists Should Order Postoperative Troponins}

The CCS recommends obtaining daily troponin measurement for 48-72 hours after surgery in patients whose risk of cardiovascular death or non-fatal MI is greater than $5 \%$ (Figure 3). ${ }^{9}$ This is recommended because the majority of postoperative myocardial 


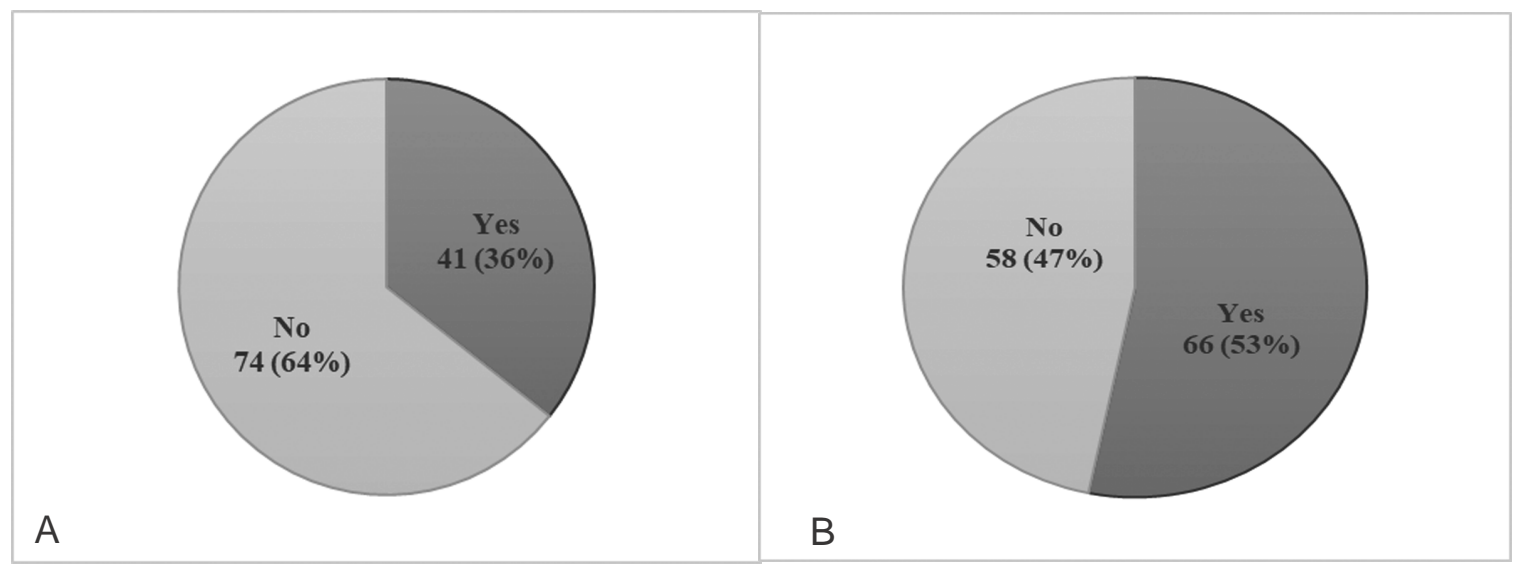

Figure 1. Survey responses from the audience at the Canadian Society of Internal Medicine plenary debate on perioperative cardiac biomarker screening. (A). You are asked to see an 81-year-old woman with high blood pressure after surgery for a hip fracture. Her blood pressure is 160/90. Would you order postoperative troponins? (B) You are asked to see an 80-year-old female before elective hip arthroplasty for arthritis. Her only past medical history is hypertension. Would you order a preoperative BNP or NT-proBNP level?

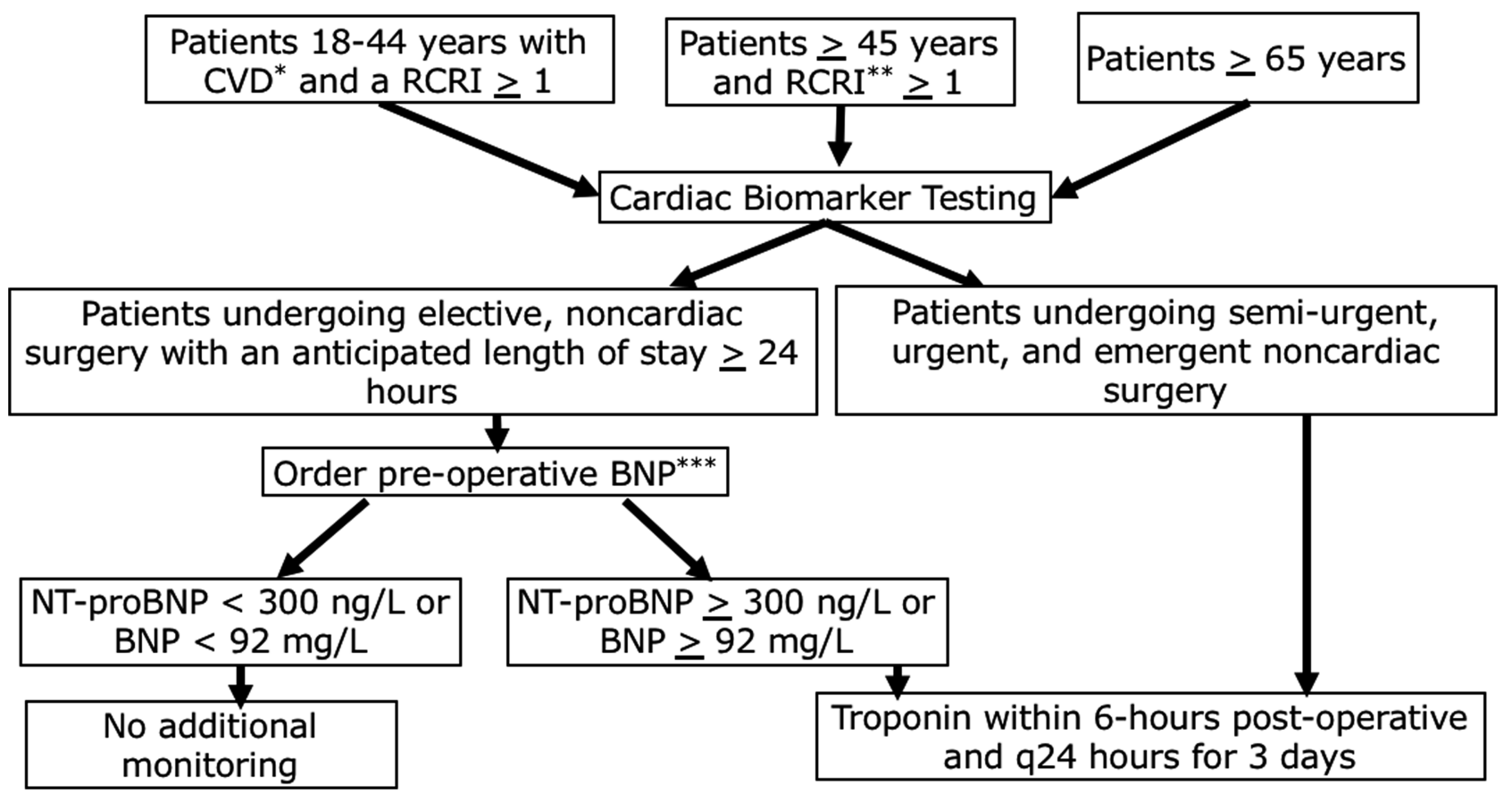

Figure 3. Canadian Cardiovascular Society guidelines algorithm for perioperative cardiac risk assessment.

${ }^{*} \mathrm{CVD}$ = cardiovascular disease; including coronary artery disease, cerebrovascular disease, peripheral arterial disease, congestive heart failure, pulmonary hypertension or severe obstructive intracardiac abnormality (for example, severe aortic or mitral stenosis).

** RCRI = revised cardiac risk index; one point for each of high-risk surgery (intraperitoneal, intrathoracic, or suprainguinal vascular surgery), coronary artery disease, congestive heart failure, cerebrovascular disease, diabetes mellitus on insulin therapy, serum creatinine greater than 177 umol/L.

${ }^{* * *} \mathrm{NP}=$ brain natriuretic peptide, referring to N-terminal pro B-type natriuretic peptide (NT-proBNP) or brain natriuretic peptide (BNP)

infarctions are asymptomatic. ${ }^{4}$ Moreover, postoperative troponin surveillance identifies patients with myocardial injury after non-cardiac surgery (MINS), who are at increased risk of postoperative complications and death. ${ }^{3,5}$ These patients have an elevated troponin resulting from myocardial injury, and most will not have ischemic symptoms or electrocardiogram changes. Identifying patients with MINS is important because enhanced post-operative care, ASA, and statins improve survival in this group. ${ }^{4,10}$ Dabigatran therapy may also improve vascular outcomes in patients with MINS. ${ }^{11}$ 


\section{Elevated Troponins Identify High-Risk Patients}

Multiple cohort studies have found that MINS occurs in 8-18\% of patients who undergo non-cardiac surgery. ${ }^{4,5,12}$ Nearly one in ten patients with a postoperative high-sensitivity troponin greater than $65 \mathrm{ng} / \mathrm{L}$ (normal < $14 \mathrm{ng} / \mathrm{L}$ ) will die within 30-days of surgery compared to less than one percent of patients with normal troponin levels. ${ }^{12}$ Increased 30 -day mortality is also seen with elevations in $4^{\text {th }}$ generation troponin $\mathrm{T}$; the mortality rate for patients with a level between $0.03-0.30 \mathrm{ng} / \mathrm{mL}$ is $9 \%$ compared to $1 \%$ for patients with a troponin $\mathrm{T} \leq 0.01 \mathrm{ng} / \mathrm{mL}$. $^{3}$ This increased risk of death extends to a full year after surgery; the one-year mortality rate after MINS is $22.5 \%$ compared to $9.5 \%$ for high-risk patients without MINS. ${ }^{12}$ These studies demonstrate a dose-response relationship between postoperative troponin elevation and 30-day mortality; the higher the peak postoperative troponin value, the greater the patient's risk of death. ${ }^{4,5}$

\section{Most Patients with MINS are Asymptomatic}

Despite the poor prognosis associated with MINS, the majority of patients with MINS are asymptomatic. The VISION cohort study included approximately 40,000 patients undergoing noncardiac surgery and found that approximately $90 \%$ of patients who met diagnostic criteria for MINS did not experience ischemic symptoms. ${ }^{5}$ Similarly, a cohort study of 2,018 highrisk surgical patients identified that over $80 \%$ of patients who had a postoperative myocardial injury were asymptomatic. ${ }^{12}$ The MANAGE trial enrolled 1,754 patients with MINS and found that $90 \%$ had no symptoms. ${ }^{11}$ In this study, $20 \%$ of patients met the universal definition for MI, of whom $46 \%$ were asymptomatic. ${ }^{11}$ The lack of symptoms in patients with MINS may be due to distracting surgical pain, postoperative delirium, the use of analgesic medications, or under recognition of atypical ischemic symptoms such as nausea. Without postoperative troponin surveillance, these high-risk patients would not be identified.

\section{Medical Therapy Improves Outcomes in MINS}

Identifying patients with postoperative myocardial injury is important because multiple interventions improve outcomes for these patients (Table 1). Post-hoc analysis of the POISE trial demonstrated that patients with postoperative MI who were prescribed ASA had significantly lower 30-day mortality (aOR $0.54,95 \%$ CI $0.29-0.99) .{ }^{4}$ The reduction in 30-day mortality was even greater in patients who were prescribed a statin (aOR $0.26,95 \%$ CI $0.13-0.54$ ). These results suggest a role for vascular protection in patients with MINS, and serve as a basis for the CCS recommendations to initiate ASA and statin therapy in this group.

Many patients who experience MINS are not on cardioprotective medications at the time of surgery. A case-control study of 667 vascular surgery patients with MINS found that about 20\% were not on antiplatelet therapy and over $40 \%$ were not taking a statin. ${ }^{10}$ In this study, patients who had their cardiac medications intensified after a MINS event had fewer major adverse cardiac events than those who did not. Though these data are from observational studies, they suggest that vascular risk reduction strategies improve postoperative outcomes. More recently, a placebo-controlled, randomized trial of 1,454 patients found that patients who received dabigatran had fewer vascular events without an increase in bleeding events. ${ }^{11}$

There is also evidence for enhancing other aspects of postoperative medical care in high-risk patients. A singlecentre study evaluated the impact of a bundled intervention on postoperative complications. ${ }^{13}$ The bundle included intensive postoperative vital sign monitoring, strict glycemic control and volume management, avoidance of hypoxia and anemia, early resumption of antiplatelet therapy, and universal venous thromboembolism prophylaxis. The investigators reported that MINS events decreased by more than half (3.9\% from $8.9 \%$ ), major adverse cardiac events decreased four-fold ( $8.1 \%$ to $1.9 \%)$ and 1-year mortality rates improved significantly ( $8.3 \%$ to $4.4 \%)$ following the implementation of the bundle. This study reinforces that high-risk patients benefit from additional nursing and physician attention and emphasizes the role in postoperative troponin surveillance for identifying these individuals.

\section{Counterpoint: Internists Should not Routinely Measure Postoperative Troponins}

The controversy surrounding postoperative troponin surveillance is reflected in the conflicting recommendations of the major cardiovascular societies. In contrast to the CCS, the AHA and the European Society of Cardiology (ESC) provide weak recommendations for postoperative troponin monitoring and in high-risk patients. ${ }^{8,14}$ Limitations of postoperative troponin surveillance include the non-specific risk associated with a postoperative troponin elevation, lack of consensus definition of postoperative myocardial injury, limited evidence for managing the postoperative myocardial injury, and the costs associated with postoperative troponin surveillance.

\section{Elevated Postoperative Troponins are a Non- Specific Marker of Risk}

Postoperative myocardial injury arises from the complex interplay of patient, surgical, and anesthetic risk factors. ${ }^{15}$ Patient-specific risk factors include prior stroke, peripheral arterial disease, coronary artery disease, congestive heart failure, diabetes, and hypertension. ${ }^{16}$ Intraoperatively and postoperatively, myocardial injury may be precipitated by hypotension, ${ }^{17}$ hypoxemia ${ }^{18,19}$ bleeding, ${ }^{20}$ and myocardial ischemia. ${ }^{21-23}$ While earlier studies suggested 
Table 1. Studies Evaluating Interventions for Postoperative Myocardial Injury or Infarction

\begin{tabular}{|c|c|c|c|c|c|}
\hline Study & Design & $\begin{array}{l}\text { Population } \\
\text { (sample size) }\end{array}$ & Intervention & Outcome & $\begin{array}{l}\text { Relative effect } \\
(95 \% \mathrm{Cl})\end{array}$ \\
\hline Ausset et al. (2010)13 & Pretest-posttest & $\begin{array}{l}\text { Orthopedic surgery } \\
(n=328)\end{array}$ & $\begin{array}{l}\text { Postoperative care } \\
\text { bundle }\end{array}$ & $\begin{array}{l}\text { Major adverse } \\
\text { cardiac events }\end{array}$ & $\begin{array}{l}\text { HR } 4.5 \\
(1.1-17.4)^{*}\end{array}$ \\
\hline $\begin{array}{l}\text { Devereaux et al. } \\
\text { (2011)4 }\end{array}$ & Prospective Cohort & $\begin{array}{l}\text { Mixed non-cardiac } \\
(\mathrm{n}=8351)\end{array}$ & $\begin{array}{l}\text { ASA } \\
\text { Statins }\end{array}$ & 30-day mortality & $\begin{array}{l}\text { aOR } 0.54 \\
(0.29-0.99) \\
\text { aOR } 0.26(0.13-0.54)\end{array}$ \\
\hline $\begin{array}{l}\text { Fourcrier et al. } \\
\text { (2014)10 }\end{array}$ & Case-control & $\begin{array}{l}\text { Vascular surgery } \\
(n=667)\end{array}$ & $\begin{array}{l}\text { Cardiovascular } \\
\text { medication } \\
\text { intensification }\end{array}$ & 1-year cardiac events & $\begin{array}{l}\text { HR } 2.80 \\
(1.05-24.2)^{* *}\end{array}$ \\
\hline $\begin{array}{l}\text { Devereaux et al. } \\
\text { (2018)11 }\end{array}$ & $\begin{array}{l}\text { Randomized controlled } \\
\text { trial }\end{array}$ & $\begin{array}{l}\text { Mixed non-cardiac } \\
(\mathrm{n}=1754)\end{array}$ & Dabigatran & $\begin{array}{l}\text { Major vascular } \\
\text { complications }\end{array}$ & HR $0.72(0.55-0.93)$ \\
\hline
\end{tabular}

*Pre-intervention vs. post-intervention; * Patients without cardiac medication intensifications vs. those with medication intensification.

that plaque rupture is common in postoperative $\mathrm{MI},{ }^{21,22}$ a recent prospective cohort study using optical coherence tomography suggests plaque rupture is rare in this setting. ${ }^{23}$ Myocardial injury can also be precipitated by non-ischemic etiologies, including sepsis, pulmonary embolus, and tachyarrhythmia. ${ }^{5}$

The complex pathophysiology of postoperative myocardial injury suggests that patients with elevated postoperative troponins are at risk of a broad range of complications. This is supported by the findings of the VISION cohort study, which reported that vascular and non-vascular death occurs with similar frequency among patients with elevated postoperative troponins. ${ }^{3}$ Thus, while increased postoperative troponins are associated with an increased risk of death, they provide little insight into the mechanism of myocardial injury, the specific complications that a patient is at an increased risk of, and which risk reduction strategies to employ.

\section{Lack of Consensus Definition of Myocardial Injury}

Several definitions of postoperative myocardial injury have been proposed (Table 2). Differences between these definitions complicate the interpretation of postoperative troponin levels. First, each of these definitions was derived using a different troponin assay. The validity of MINS diagnostic criteria, when applied to troponin assays not evaluated in the original studies, has not been established. Second, the relevance of preoperative troponin values varies between definitions of postoperative myocardial injury. The MINS definitions derived from the VISION cohort study is based on postoperative troponin values only. ${ }^{3,5}$ In a more recent study, postoperative myocardial injury was defined as an elevated postoperative troponin in comparison to preoperative measurements. ${ }^{12}$ More than half of the patients in this study had an elevated preoperative troponin level. These data suggest that many postoperative myocardial injuries diagnosed using postoperative TROPONIN values may only have elevated troponin that existed prior to surgery, not due to surgery. These patients likely have a worse prognosis irrespective of whether they have surgery or not. Finally, the prognostic implications vary substantially between these different criteria (Table 2). While the negative predictive values are similar for all three definitions of myocardial injury, the positive predictive values range from $4-10 \%{ }^{11,16}$

The lack of consensus definition for postoperative myocardial injury has implications for patients, healthcare providers, and healthcare institutions. Patients cared for at centres where hs-TnT assays are used are more likely to be diagnosed with MINS than patients admitted to institutions where less sensitive assays are being used. These patients are also more likely to be exposed to additional testing and treatments, many of which are of unproven benefit. Given that the incidence of postoperative myocardial injury varies two-fold depending on the criteria used, ${ }^{11,16}$ it can be anticipated that the workload of clinicians providing postoperative medical care will vary drastically, as will the costs incurred by healthcare institutions.

\section{Optimal Management of Post-operative Myocardial Injury is Unclear}

Observational studies suggest that ASA and statins improve outcomes in patients with MINS. ${ }^{4}$ However, these studies have significant limitations. First, while post-hoc analysis of the POISE trial data shows that ASA and statin use is associated with decreased mortality in patients with postoperative myocardial 
Conference Proceedings

Table 2. Diagnostic Criteria for Myocardial Injury

\begin{tabular}{|c|c|c|c|c|c|}
\hline Author (Year) & Troponin Assay & MINS definition & Incidence & PPV & NPV \\
\hline $\begin{array}{l}\text { Botto et al. } \\
\text { (2014)16 }\end{array}$ & $\begin{array}{l}\text { Troponin T } \\
\text { (4th generation) }\end{array}$ & $\begin{array}{l}\text { Peak postoperative troponin } \geq 0.03 \mu \mathrm{g} / \mathrm{L} \text { judged due to } \\
\text { myocardial ischemia }\end{array}$ & $8.0 \%$ & $9.8 \%$ & $98.9 \%$ \\
\hline $\begin{array}{l}\text { Devereaux et al. } \\
(2017) 5\end{array}$ & hs-Troponin T & $\begin{array}{l}\text { Elevated postoperative troponin ( } 20-65 \mathrm{ng} / \mathrm{L} \text { with } \\
\text { a change of } 5 \mathrm{ng} / \mathrm{L} \text { or } \geq 65 \text { ) judged as resulting from } \\
\text { myocardial ischemia }\end{array}$ & $17.9 \%$ & $4.1 \%$ & $99.4 \%$ \\
\hline $\begin{array}{l}\text { Puelacher et al. } \\
\text { (2018)12 }\end{array}$ & hs-Troponin T & $\begin{array}{l}\text { Increase in troponin of } \geq 14 \mathrm{ng} / \mathrm{L} \text { above preoperative } \\
\text { measurement }\end{array}$ & $16.0 \%$ & $9.8 \%$ & $98.4 \%$ \\
\hline
\end{tabular}

MINS = myocardial injury after non-cardiac surgery; NPV = negative predictive value; PPV = pulse pressure variation.

infarction, ${ }^{4}$ the generalizability of these findings to the broader MINS population remains unknown. Second, the magnitude of the benefit of statins for treating postoperative MI observed in the POISE trial exceeds that seen in the landmark trials for acute coronary syndrome ${ }^{24,25}$ which brings the external validity of the POISE findings into question. Third, rates of preoperative use of ASA, statins, and angiotensin-converting enzyme inhibitors blockers were low in the cohort study of vascular surgery patients, ${ }^{10}$ despite these medications being indicated in this population for the prevention of cardiovascular events. ${ }^{26}$ The benefits seen with medication intensification may, therefore, reflect the appropriate use of secondary prevention for peripheral arterial disease rather than their impact on postoperative MI. Finally, both studies had observational designs and should, therefore, be considered hypothesis-generating but not definitive evidence.

More recently, the MANAGE trial evaluated dabigatran in patients with MINS. ${ }^{11}$ Dabigatran $110 \mathrm{mg}$ twice daily was associated with a significant reduction in the composite primary outcome of major vascular complications (vascular mortality, non-fatal MI, non-hemorrhagic stroke, peripheral arterial thrombosis, amputation, and symptomatic venous thromboembolism) and was non-inferior to placebo for the composite primary safety outcome of life-threatening, major, and critical organ bleeding. However, recruitment was slower than anticipated, and as a result, the study investigators had to reduce their sample size and expand their composite primary outcome. This suggests that dabigatran is unacceptable to many patients and healthcare providers for the treatment of MINS. Forty-six percent of patients prematurely discontinued the drug and the median duration of use was only 40 days. The safety of dabigatran in this setting, therefore, remains uncertain. In terms of effectiveness, the only component of the composite primary outcome that was significantly reduced was non-hemorrhagic stroke. Given these limitations, additional research is needed before dabigatran or other direct oral anticoagulants can be routinely used for the treatment of postoperative myocardial injury.

The availability of effective treatment has been argued to be the most important criteria for evaluating screening programs. ${ }^{27}$ As discussed above, studies evaluating interventions in MINS had significant methodological limitations. For this reason, troponin surveillance is not justified in most patients undergoing non-cardiac surgery.

\section{Postoperative Troponin Surveillance is Costly}

At face value, post-operative troponin surveillance appears to be a low-cost intervention. The cost of troponin monitoring is approximately 4-7 dollars per test or 9-20 dollars for three days of testing. ${ }^{28}$ However, the downstream costs are substantial. A recent prospective cohort study of higher-risk surgical patients routinely measured troponins postoperatively. ${ }^{12}$ Sixteen percent of patients fulfilled the definition of postoperative myocardial injury, of which $52 \%$ went on to have a cardiology consultation, $22 \%$ had an echocardiogram, and $8 \%$ had an angiogram performed. Additional costs include medications used for the treatment of MINS and patient follow-up. Thus, postoperative troponin monitoring results in substantial downstream resource use, which in turn may increase healthcare expenditures and decrease access to these resources for non-surgical patients.

\section{Point: Internists Should use Natriuretic Peptide Testing for Perioperative Cardiac Risk Assessment}

Several prospective cohort studies and meta-analyses demonstrated an association between preoperative natriuretic peptide levels (NP) levels and postoperative cardiac complications. ${ }^{29,30}$ These findings led the CCS to make a strong recommendation for preoperative testing of N-terminal B-type natriuretic peptide (NT-proBNP) or brain natriuretic peptide (BNP) levels in patients with risk factors for cardiovascular complications (i.e. $\geq 65$ years of age, $\geq 45$ 
years of age with significant cardiovascular disease, or a Revised Cardiac Risk Index $\geq 1$ ). ${ }^{9}$ In contrast, the AHA recommends using clinical risk indices and functional assessment for predicting patients' risk of postoperative cardiovascular complications, with adjunct cardiac testing in certain circumstances. ${ }^{8}$ Natriuretic peptide (referring to either NT-proBNP or BNP) testing offers important advantages over this approach; it improves clinical risk prediction compared with functional assessment and is less expensive than pharmacologic stress testing.

\section{Functional Assessment Cannot Identify High-Risk Patients}

The AHA perioperative guidelines recommended using subjective functional assessment to assess patients' risk of perioperative cardiovascular complications. ${ }^{8}$ Patients who, by history, were able to exert four metabolic equivalents (METs) were deemed to be at low risk of postoperative death or MI and did not require further testing. This recommendation was based on the results of a prospective cohort study demonstrating that poor exercise tolerance is associated with an increased risk of myocardial ischemia and a non-significant trend towards an increased risk of total cardiovascular events. ${ }^{31}$ However, recent evidence suggests that functional capacity, though intuitively important, is ineffective for risk-stratification. The METS study included 1,401 patients with cardiovascular risk factors and compared subjective functional assessment with cardiopulmonary exercise testing (CPET) for predicting postoperative death or complications. ${ }^{32}$ Subjective functional assessment had a sensitivity of $19.2 \%$ and a specificity of $94.7 \%$ to identify patients with an exercise capacity less than 4 METs when measured by CPET. Importantly, regardless of the method used, exercise capacity did not correlate with any post-operative outcome, including 30-day death or MI.

\section{Pharmacologic Stress Testing Can Cause Harm in Low-Risk Patients}

The AHA guidelines recommend pharmacologic stress testing for patients with poor functional capacity in whom testing would alter management. ${ }^{8}$ In support of this recommendation, metanalyses of studies evaluating preoperative myocardial perfusion imaging demonstrated that large areas of inducible myocardial ischemia are associated with increased risk of postoperative cardiac complications. ${ }^{2,33}$ These results should be interpreted with caution as the majority of the included studies were retrospective in nature and many were at risk of ascertainment bias. More recently, a Canadian cohort study of more than 271,000 patients found that patients who underwent pharmacologic stress testing had lower postoperative mortality compared to patients who underwent no testing. ${ }^{34}$ However, subgroup analysis suggested that in low-risk patients (RCRI
0 ), pharmacologic stress testing was associated with increased mortality (hazard ratio 1.35, 95\% CI 1.05-1.74), indicating that careful selection of patients who would benefit is critical. ${ }^{34}$ Despite this, rates of low-value preoperative pharmacologic testing are known to be very high; a review of 501 preoperative stress tests found that two-thirds were not appropriate and $74 \%$ were performed in low-risk patients. ${ }^{35}$ Further analysis suggests that the reduced mortality seen with pharmacologic stress testing was not driven by revascularization, but may have been attributable to increased postoperative monitoring and pharmacologic management of known cardiovascular disease risk factors. ${ }^{34}$ Importantly, the number needed to treat for pharmacologic stress testing to avoid one death was 221, and one-third of the MIs and deaths in the study occurred in patients who had a normal preoperative stress test. $^{32}$ For these reasons, preoperative pharmacologic stress testing is not appropriate for the majority of patients undergoing non-cardiac surgery.

\section{Natriuretic Peptide Testing Enhances Preoperative Cardiac Risk Assessment}

Unlike self-reported exercise tolerance, preoperative NP levels predicted postoperative cardiac complications in multiple prospective cohort studies. A systematic review and metaanalysis determined that an elevated preoperative NP level had an adjusted OR of 19.3 (95\% CI 8.5-43.7) for 30-day cardiac complications. ${ }^{30}$ Subsequently, an individual patient-level meta-analysis of 18 prospective cohort studies, which included 2,179 patients undergoing a variety of surgeries, reported that patients with an elevated preoperative NP level (NT-proBNP $\geq 300 \mathrm{ng} / \mathrm{L}$ or BNP $\geq 92$ ) had a $21.8 \%$ risk of MI and death at 30 -days compared with a $4.9 \%$ risk in patients with NP levels below these thresholds. ${ }^{36}$ Based on these analyses, the CCS guidelines recommend obtaining preoperative NP measurements in patients with a baseline risk $>5 \%$ for postoperative death or non-fatal MI to enhance risk assessment. The results of the METS trial also confirmed the prognostic value of preoperative NP measurements. An elevated preoperative NP level was associated with an increased risk of death or MINS at 30-days (aOR 1.78, $95 \% \mathrm{CI} 1.21-2.62)$ and death at one-year postoperatively (aOR $2.91,95 \%$ CI $1.54-5.49){ }^{32}$

\section{Counterpoint: Internists Should Not Use NP Levels for Preoperative for Cardiac Risk Assessment}

Preoperative assessment using NP measurement is controversial (see Figure 1). In contrast to the CCS, the ESC provided a weak recommendation for preoperative NP measurement in highrisk patients and the AHA did not provide a recommendation addressing preoperative NP measurement. ${ }^{8,14}$ Natriuretic peptide levels should not be measured preoperatively given the modest 
incremental prediction provided by NP levels, the uncertain impact of NP testing on clinical outcomes, and practical barriers to implementing preoperative NP testing in clinical practice.

\section{Prediction Provided by NP Levels is Modest}

The prognostic value of preoperative NP levels has been evaluated in several small, prospective cohort studies. A systematic review and meta-analysis identified 9 studies that evaluated the predictive performance of preoperative NP levels in 3,281 patients. ${ }^{30}$ The results of the study suggested a strong association between preoperative NP levels and postoperative cardiovascular complications (aOR 19.3, 95\% CI 8.5-43.7). However, the pooled outcome data used in this metanalysis was based on study-specific NP thresholds. This would have overestimated the association between preoperative NP levels and postoperative cardiovascular complications. ${ }^{37}$

A subsequent individual patient metanalysis of 2,179 patients undergoing non-cardiac surgery applied a single BNP and NT-proBNP threshold across the entire dataset. The association between preoperative NP level and postoperative cardiovascular complications was less compelling in this study (aOR 3.40, 95\% CI 1.81-3.96). Moreover, NP levels provided only fair discrimination for this outcome (AUC 0.71 for BNP; 0.69 for NT-proBNP). ${ }^{30}$ The authors also assessed the net reclassification for a model that included baseline variables (age, surgery type, surgery urgency, RCRI score) and NP levels compared with a model that included baseline variables alone. After accounting for patients who were incorrectly reclassified, the model that incorporated NP levels correctly reclassified only $16 \%$ of patients into a higher or lower risk category. Moreover, the majority of patients who were correctly reclassified had their risk-adjusted by a single 5\% risk category. This suggests that the additional prediction provided by preoperative NP levels is modest at best.

The recent METS study also evaluated the association between preoperative NT-proBNP levels and postoperative cardiovascular complications. ${ }^{32}$ Preoperative NT-proBNP levels did not predict the primary outcome of death or MI at 30-days after surgery (aOR 1.88, 95\% CI 0.89-3.96), which brings into question the utility of preoperative NP levels for predicting short-term cardiovascular complications. However, preoperative NT-proBNP levels were associated with one-year mortality, as discussed earlier in this review.

\section{The Impact of BNP Testing on Patient Outcomes is Uncertain}

The AHA recommends a 6-step process for evaluating novel biomarkers, which includes performing proof of concept studies, conducting prospective validation studies, assessing incremental prediction, evaluating clinical utility, assessing impact on clinical outcomes, and establishing cost-effectiveness. ${ }^{38}$ Studies to date have prospectively validated preoperative NP testing for cardiac risk assessment and demonstrated incremental improvement in prediction compared with clinical risk factors. ${ }^{30,36}$ However, no studies have evaluated the impact of preoperative NP testing on care delivery or patient outcomes. These studies should be performed before widely implementing preoperative NP testing.

\section{Practical Barriers to Preoperative BNP Testing are Substantial}

Implementing preoperative NP testing into preoperative risk assessment poses substantial challenges for both healthcare institutions and front-line providers. First, many healthcare providers do not have access to NP testing. A poll of CSIM annual meeting attendees revealed that $40 \%$ did not have access to these laboratory tests at their institution (Figure 4). Point of care testing is a reasonable alternative in these settings, but will nevertheless require institutions to purchase the necessary equipment and reagents. Second, the optimal timing of preoperative NP testing has not been established and has varied in prospective studies conducted to date. ${ }^{30}$ Third, studies to date have not evaluated how obesity and renal failure impact the prognostic performance of preoperative NP levels. Fourth, a clinical risk calculator that incorporates both clinical risk factors (e.g. age, surgery type, RCRI score, etc.) and NP levels are yet to be developed for clinical use. This is necessary as the prediction provided by preoperative NP levels is incremental to that provided by clinical risk factors and indices.

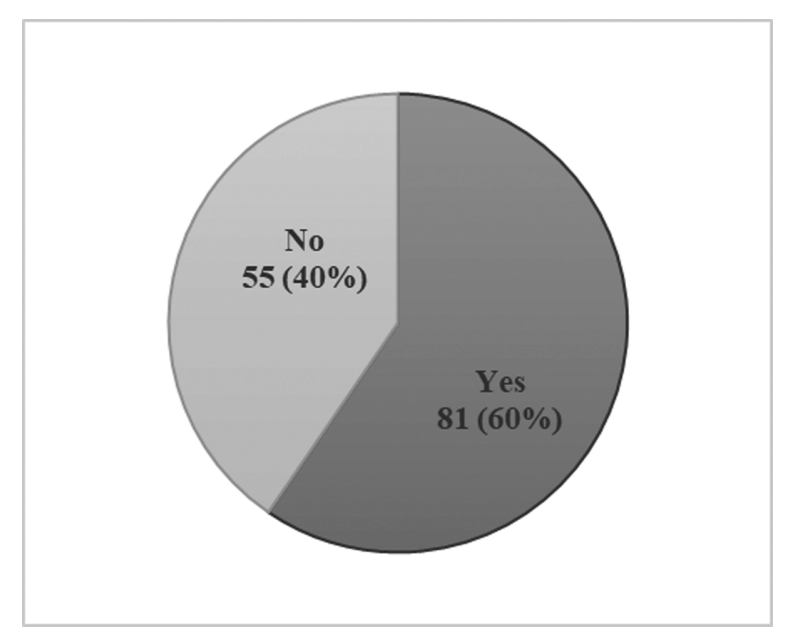

Figure 4. Survey responses for audience members at the Canadian Society of Internal Medicine annual meeting plenary debate on perioperative cardiac biomarker surveillance for the question; Do you have same-day access to natriuretic peptide testing at your centre? 


\section{Conclusion}

Cardiac biomarkers show promise for enhancing perioperative cardiac risk assessment. Preoperative NP levels provide incremental prediction for postoperative cardiovascular complications while postoperative troponin surveillance facilitates early identification of patients with postoperative myocardial injury. There is potential to improve outcomes in these patients by initiating cardioprotective medications and enhancing postoperative medical care. However, the incremental risk prediction provided by NP testing is modest and most evidence for treating postoperative myocardial injury is from observational studies. It is also unknown whether the implementation of preoperative NP testing and postoperative troponin testing will improve clinical outcomes. We recommend that general internists and institutions take into consideration local availability of NP testing, capacity for postoperative medical follow-up, and patients' preferences when utilizing these novel perioperative risk assessment modalities.

\section{References}

1. Canadian Institute of Health Information. Inpatient hospitalizations, surgeries and newborn indicators, 2016-2017. 2018.

2. Beattie WS, Abdelnaem E, Wijeysundera DN, and Buckley DN. A metaanalytic comparison of preoperative stress echocardiography and nuclear scintigraphy imaging. Anesth Analg 2006;102:8-16.

3. Devereaux P, Chan M, Alonso-Coello P, et al. Association between postoperative troponin levels and 30-day mortality among patients undergoing non-cardiac surgery. JAMA 2012;307:2295-304.

4. Devereaux P, Xavier D, Pogue J, et al. Characteristics and short-term prognosis of perioperative $\mathrm{MI}$ in patients undergoing non-cardiac surgery a cohort study. Ann Intern Med 2011;154:523-8.

5. Devereaux P, Biccard BM, Sigamani A, et al. Association of postoperative high-sensitivity troponin levels with myocardial injury and 30-day mortality among patients undergoing non-cardiac surgery. JAMA 2017;317:1642-51.

6. Smilowitz NR, Gupta N, Ramakrishna H, Guo Y, Berger JS, Bangalore S. Perioperative major adverse cardiovascular and cerebrovascular events associated with non-cardiac surgery. JAMA Cardiol2017;2:181-7.

7. Smilowitz NR, Beckman JA, Sherman SE, Berger JS. Hospital readmission after perioperative acute MI associated with non-cardiac surgery. Circulation 2018;137:2332-9.

8. Fleisher LA, Fleischmann KE, Auerbach AD, et al. 2014 ACC/AHA guideline on perioperative cardiovascular evaluation and management of patients undergoing non-cardiac surgery: a report of the American College of Cardiology/American Heart Association Task Force on practice guidelines. J Am Coll Cardiol 2014;64:e77-137.

9. Duceppe E, Parlow J, MacDonald P, et al. Canadian Cardiovascular Society Guidelines on Perioperative Cardiac Risk Assessment and Management for Patients Who Undergo Non-cardiac Surgery. Can J Cardiol 2017;33:17-32.

10. Foucrier A, Rodseth R, Aissaoui M, et al. The long-term impact of early cardiovascular therapy intensification for postoperative troponin elevation after major vascular surgery. Anesth Analg 2014;119:1053-63.

11. Devereaux PJ, Duceppe E, Guyatt G, et al. Dabigatran in patients with myocardial injury after non-cardiac surgery (MANAGE): an international, randomised, placebo-controlled trial. Lancet 2018;391:2325-34.

12. Puelacher C, Lurati Buse G, Seeberger D, et al. Perioperative Myocardial Injury After Non-cardiac Surgery: Incidence, Mortality, and Characterization. Circulation 2018;137:1221-32.
13. Ausset S, Auroy Y, Verret C, et al. Quality of postoperative care after major orthopedic surgery is correlated with both long-term cardiovascular outcome and troponin Ic elevation. Anesthesiology 2010;113:529-40.

14. Kristensen SD, Knuuti J, Saraste A, et al. 2014 ESC/ESA Guidelines on noncardiac surgery: cardiovascular assessment and management. Eur Heart J 2014;35:2383-431.

15. Devereaux P, Sessler DI. Cardiac complications in patients undergoing major non-cardiac surgery. N Engl J Med 2015;373:2258-69.

16. Botto F, Alonso-Coello P, Chan MT, et al. Myocardial injury after noncardiac surgery: a large, international, prospective cohort study establishing diagnostic criteria, characteristics, predictors, and 30-day outcomes. Anesthesiology 2014;120:564-78.

17. van Waes JA, Van Klei WA, Wijeysundera DN, et al. Association between intraoperative hypotension and myocardial injury after vascular surgery. Anesthesiology 2016;124:35-44.

18. Gill N, Wright B, Reilly C. Relationship between hypoxaemic and cardiac ischaemic events in the perioperative period. Br J Anaesth 1992;68:471-3.

19. Goldman M, Reeder M, Muir A, et al. Repetitive nocturnal arterial oxygen desaturation and silent myocardial ischemia in patients presenting for vascular surgery. J Am Geriatr Soc 1993;41:703-9.

20. Kamel H, Johnston SC, Kirkham JC, et al. Association between major perioperative hemorrhage and stroke or Q-wave MI. Circulation 2012; 126:207-12.

21. Gualandro DM, Campos CA, Calderaro D, et al. Coronary plaque rupture in patients with MI after non-cardiac surgery: frequent and dangerous. Atherosclerosis 2012;222:191-5.

22. Dawood MM, Gutpa DK, Southern J, et al. Pathology of fatal perioperative MI: implications regarding pathophysiology and prevention. Int J Cardiol 1996;57:37-44.

23. Sheth $T$, Natarajan $M, H$ sieh $V$, et al. Incidence of thrombosis in perioperative and non-operative MI. Br J Anaesth 2018;120:725-33.

24. Schwartz GG, Olsson AG, Ezekowitz MD, et al. Effects of atorvastatin on early recurrent ischemic events in acute coronary syndromes: the MIRACL study: a randomized controlled trial. JAMA 2001;285:1711-8.

25. Cannon $\mathrm{CP}$, Braunwald $\mathrm{E}, \mathrm{McCabe} \mathrm{CH}$, et al. Intensive versus moderate lipid lowering with statins after acute coronary syndromes. N Engl J Med 2004;350:1495-504.

26. Gerhard-Herman MD, Gornik HL, Barrett C, et al. 2016 AHA/ACC guideline on the management of patients with lower extremity peripheral artery disease: executive summary. J Am Coll Cardiol 2017;69:1465-508.

27. Wilson JMG, Jungner G, Organization WH. Principles and practice of screening for disease. World Health Organization: Geneva; 1968.

28. Canadian Agency for Drugs and Technologies in Health. High-sensitivity cardiac troponin for the rapid diagnosis of acute coronary syndrome in the emergency department: a clinical and cost-effectiveness evaluation. 2013.

29. Rodseth R, Padayachee L, Biccard BM. A meta-analysis of the utility of preoperative brain natriuretic peptide in predicting early and intermediate-term mortality and major adverse cardiac events in vascular surgical patients. Anaesthesia 2008;63:1226-33.

30. Karthikeyan G, Moncur RA, Levine O, et al. Is a preoperative brain natriuretic peptide or $\mathrm{N}$-terminal pro-B-type natriuretic peptide measurement an independent predictor of adverse cardiovascular outcomes within 30 days of non-cardiac surgery?: A systematic review and metaanalysis of observational studies. J Am Coll Cardiol 2009;54:1599-606.

31. Reilly DF, McNeely MJ, Doerner D, et al. Self-reported exercise tolerance and the risk of serious perioperative complications. Arch Intern Med 1999;159:2185-92.

32. Wijeysundera DN, Pearse RM, Shulman MA, et al. Assessment of functional capacity before major non-cardiac surgery: an international, prospective cohort study. Lancet 2018;391:2631-40.

33. Etchells E, Meade M, Tomlinson G, Cook D. Semiquantitative dipyridamole myocardial stress perfusion imaging for cardiac risk assessment before noncardiac vascular surgery: a metaanalysis. J Vasc Surg 2002;36:534-40. 
34. Wijeysundera DN, Beattie WS, Austin PC, Hux JE, Laupacis A. Non-invasive cardiac stress testing before elective major non-cardiac surgery: population based cohort study. Br Med J 2010;340.

35. Peterson B, Ghahramani M, Emerich M, Foy AJ. Frequency of appropriate and low-risk non-cardiac preoperative stress testing across medical specialties. Am J Cardiolol 2018;122:744-8.

36. Rodseth RN, Biccard BM, Le Manach Y, et al. The prognostic value of preoperative and post-operative B-type natriuretic peptides in patients undergoing non-cardiac surgery: a systematic review and individual patient data meta-analysis. Journal of the Am Coll Cardiol 2014;63:170-80.

37. Potgieter D, Simmers D, Ryan L, et al. N-terminal pro-B-type natriuretic peptides' prognostic utility is overestimated in meta-analyses using studyspecific optimal diagnostic thresholds. Anesthesiology 2015;123:264-71.

38. Hlatky MA, Greenland P, Arnett DK, et al. Criteria for evaluation of novel markers of cardiovascular risk: a scientific statement from the American Heart Association. Circulation 2009;119:2408-16.

\section{Top 10 Most Downloaded Articles Over the Past Year}

1. BMJ Rapid Recommendations: A Possible Revolution in Clinical Practice Guidelines

Gordon Guyatt, Thomas Agoritsas, Lyubov Lytvyn, Reed Siemieniuk, Per Vandvik

2. Renal Limited Lupus-Like Nephritis

Jumana Amir, Salwa Sheikh, Abdulrazack Amir

3. Acute Hepatitis Associated with "Thermogenic Fat Burner" Weight Loss Supplementation: A Case Report

Marko Popovic, Maxime Billick, Matthew R. Robinson

4. Emerging Barrier to Timely Care of Hip Fracture Patients: A Prospective Study of Direct Oral Anticoagulation and Time to Surgery Marlis T Sabo, Fatima Mahdi, Marcia Clark

5. A CanMEDS Competency-Based Assessment Tool for High-Fidelity Simulation in Internal Medicine: The Montreal Internal Medicine Evaluation Scale (MIMES)

Patrice Chrétien Raymer, Jean-Paul Makhzoum, Robert Gagnon, Arielle Levy, Jean-Pascal Costa

6. Gender Equity in Academic Medicine: Why Should We Care?

Sonia S. Anand, Anita I. Anand, BA, BA, LLB, LLM

7. Liver Abscess Metastatic Syndrome Caused by Hypermucoviscous Klebsiella Pneumoniae in a Canadian Patient of Vietnamese

Origin

Sandra Patricia Criales Doria, Alexandre Lafleur, Philippe Gervais

8. Not Every Low Back Pain is a Radiculopathy

Pankaj Bansal, Laura Grennan, Karthik Mahadevappa, Emilia Semenov, Shanker Nesathurai

9. A Challenging Case of Non-resolving Pneumonia: Keeping Antisynthetase Syndrome in the Differential Diagnosis

Stephanie Lapinsky, MD, Jessica Leen, MD, Jessica Mak, BSc. Pharm (Hons), RPh. MD Candidate,

Mohammed A. Shafiee, MD

10. Anterior Spinal Artery Syndrome as Complication of Bronchial Artery Embolization

Ka Hong (Casey) Chan, Chris White, Jason K Wong 